\title{
Rapid and Economic Access to Some NSAIDS-Based Bioactive Heterocycles
}

\author{
A. V. Chavan, ${ }^{1}$ R. R. Somani, ${ }^{2}$ P. P. Kalantri, ${ }^{3}$ and D. T. Makhija ${ }^{3}$ \\ ${ }^{1}$ Department of Pharmaceutical Chemistry, C.U. Shah College of Pharmacy, S.N.D.T. Women's University, \\ Santacruz (West), Maharashtra, Mumbai 400 049, India \\ ${ }^{2}$ Department of Pharmaceutical Chemistry, V.E.S. College of Pharmacy, Chembur, Mumbai 400074, India \\ ${ }^{3}$ Department of Pharmaceutical Chemistry, Bharati Vidyapeeth's College of Pharmacy, Sector 8, C.B.D. Belapur, \\ Maharashtra, Navi Mumbai 400614, India
}

Correspondence should be addressed to R. R. Somani; rakeshrsomani@gmail.com

Received 17 June 2012; Revised 4 August 2012; Accepted 22 August 2012

Academic Editor: Bartolo Gabriele

Copyright (c) 2013 A. V. Chavan et al. This is an open access article distributed under the Creative Commons Attribution License, which permits unrestricted use, distribution, and reproduction in any medium, provided the original work is properly cited.

The use of microwave energy for the synthesis is considered as one of the alternate strategies. The present work includes the synthesis of some newer 2,5-disubstituted-1,3,4-oxadiazole analogues using both conventional and alternate rapid methods. The conventional method involves the synthesis of the target molecules by using cyclodehydrating agent like $\mathrm{POCl}_{3}$, while the other method consists of using some catalytic reagents like $\mathrm{PbO}_{2}$. Also, the short reaction time provided by microwave synthesis makes it ideal for the reaction. Hence, this project describes an efficient and operationally simple method for the microwave-assisted synthesis of some bioactive heterocycles based on NSAIDs.

\section{Introduction}

Synthetic methodology, as the building block of organic synthesis, continuously seeks for new reagents, better reaction conditions, and the usage of newer catalysts for more efficient and selective synthesis. The increasing environmental consciousness throughout the world has put a pressing need to develop an alternate synthetic approach for biologically and synthetically important compounds [1]. The best option to accelerate these synthetic processes is the use of microwave irradiation [2]. The use of Microwave-Assisted Organic Synthesis (MAOS) in synthetic chemistry reduces reaction time and increases product yield. Some of the major advances in chemistry have also been observed in the area of catalysts. Modern catalysts provide highly selective routes to synthesize chemical products [3].

Nonsteroidal anti-inflammatory drugs (NSAIDs) are essential compounds to treat sign and symptoms of inflammation and pain. They exert their effect mainly through inhibition of cyclooxygenases (COXs), the key enzyme in prostaglandin (PG) biosynthesis. But the ulcerogenic potential limits their use. The ulcerogenic nature of NSAIDs is attributed to free $-\mathrm{COOH}$ group present therein. The literature is flooded with different approaches used to modify free $-\mathrm{COOH}$ thereby modifying the activity of the parent NSAIDs [4]. In the present work, as shown in the Figure 1 , this free $-\mathrm{COOH}$ group is modified by converting it into $1,3,4$-oxadiazole ring which is toxophoric in nature and acts as bioisostere $[5,6]$.

Heterocyclic compounds are proven backbone for the discovery of many bioactive compounds. Molecules containing a 1,3,4-oxadiazole scaffold have shown broad range of biological activities like antibacterial, antitubercular, antifungal, analgesic, and anti-inflammatory properties $[7,8]$. Consequently, the synthesis of such heterocycle has attracted considerable attention and a wide variety of methods have been used for its assembly [9]. By far the most common strategy involves the dehydrative cyclization of diacylhydrazides, usually with strongly acidic reagents such as $\mathrm{POCl}_{3}$, $\mathrm{SO}_{2} \mathrm{Cl}, \mathrm{P}_{2} \mathrm{O}_{5}, \mathrm{H}_{2} \mathrm{SO}_{4}$, or PPA [10]. Unfortunately, most of the procedures which have been described for this reaction entail using toxic reagents or solvents, long reaction times, and so forth. Keeping this in view, the present work aims at the synthesis of some newer 2,5-disubstituted-1,3,4-oxadiazole 


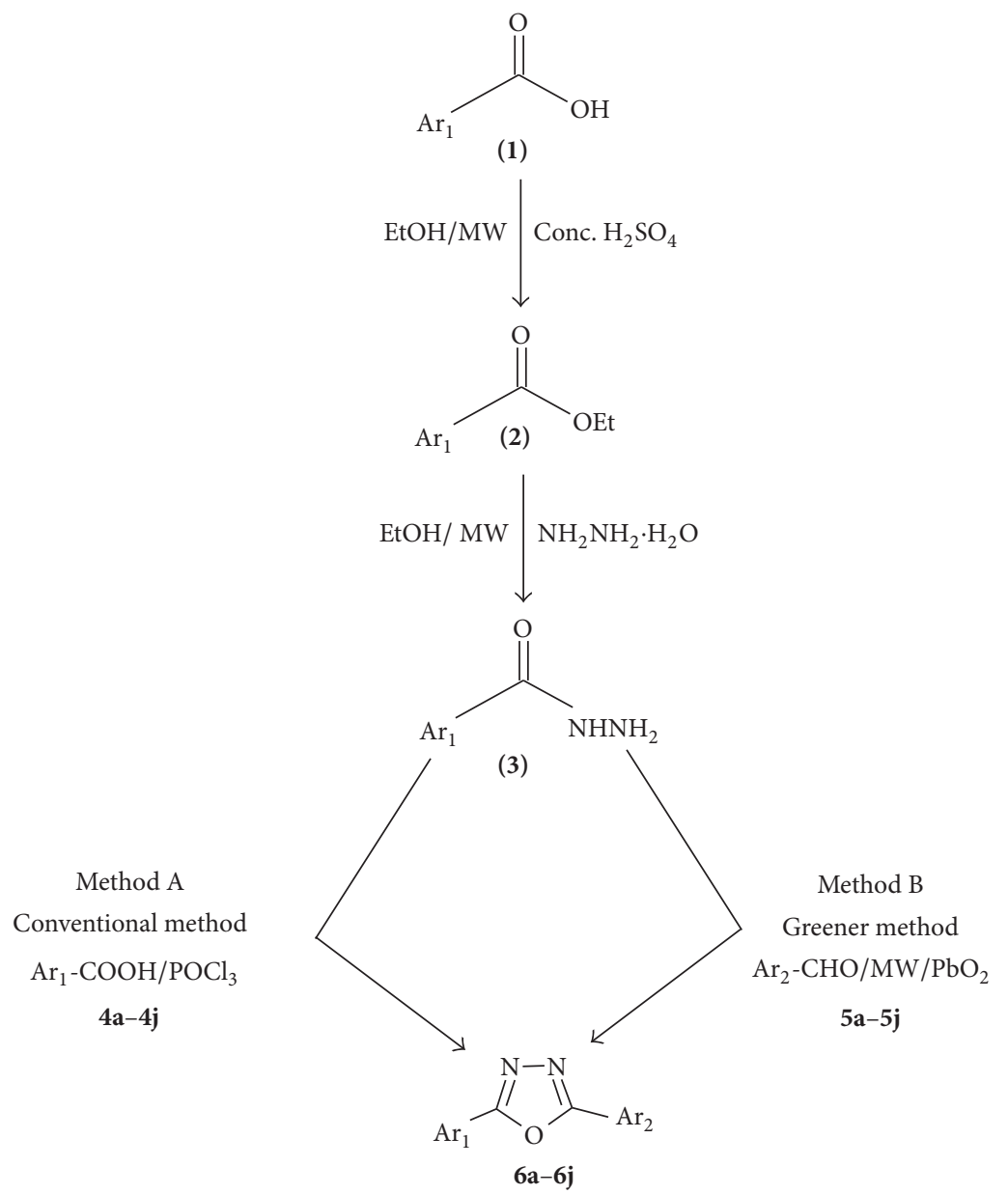

\begin{tabular}{cll}
\hline NSAIDs & Compound Number & \multicolumn{1}{c}{$\mathrm{Ar}_{2}$} \\
\hline Ibuprofen & $\mathbf{6 a}$ & Phenyl \\
derivatives & $\mathbf{6 b}$ & 4-Methylphenyl \\
Aceclofenac & $\mathbf{6 c}$ & 2-Hydroxyphenyl \\
derivatives & $\mathbf{6 d}$ & Benzyl \\
& $\mathbf{6 e}$ & 4-Pyridyl \\
\hline
\end{tabular}

FIGURE 1: Scheme of synthesis with various substituents.

analogues based on NSAIDs using both conventional and rapid alternate methods. Conventional method involves synthesis of the target molecules by using cyclodehydrating agent like $\mathrm{POCl}_{3}$, while alternate method consists of use of some newer, selective, catalytic reagents like lead oxide. Then the comparison based on the rapidness, cost-effectiveness, and ecofriendliness is done to establish the most effective method. Furthermore, the compounds were evaluated for antimicrobial and analgesic activities to check their biological potential. 


\section{The Development of a New Synthetic Method- ology for the Preparation of 2,5-Disubsti- tuted-1,3,4-oxadiazole}

\subsection{Experimental}

2.1.1. Material and Methods. Melting points were determined by open capillary method on a "Veego VMP-D" apparatus and are uncorrected. Purity of the compounds was checked on silica gel G plates of size $3 \times 8 \mathrm{~cm}$ (SigmaAldrich) and visualized by UV or in an iodine chamber. The IR spectra were recorded in the $4000-400 \mathrm{~cm}^{-1}$ range using $\mathrm{KBr}$ discs on an IR 8400 S SHIMADZU spectrometer. ${ }^{1} \mathrm{H}$ NMR spectra were recorded on Varian Mercury $(300 \mathrm{MHz})$ spectrometer in $\mathrm{CDCl}_{3}$ or $\mathrm{DMSO}-\mathrm{d}_{6}$ as solvent using trimethylsilane (TMS) as an internal reference standard and values are expressed in ppm. Microwave synthesis was carried out using modified catalyst-type microwave oven, at power level ranging from 1 to 9 at 140-700 watt. Spectral analysis was recorded at Sophisticated Analytical Instrument Facility (SAIF), IIT, Mumbai, and the Institute of Science, Mumbai. Column chromatography was done for purification of compounds using appropriate solvent system. Ibuprofen and Aceclofenac were procured from Nicholas Piramal Pvt. Ltd., Mumbai, as gift samples.

2.1.2. Synthesis of Esters [11]. The mixture of NSAID (0.1 mole), absolute ethanol ( 0.7 mole), and catalytic amount of conc. $\mathrm{H}_{2} \mathrm{SO}_{4}(5 \mathrm{~mL})$ was refluxed for about 180 minutes at 560 watt in microwave oven. The reaction mixture was cooled and poured with stirring on crushed ice. It was then neutralized with $10 \%$ sodium bicarbonate solution and extracted with $5 \times 25 \mathrm{~mL}$ of diethyl ether. Combined ethereal extracts were dried over anhydrous $\mathrm{MgSO}_{4}$ and distilled to yield the desired products $(2 \mathbf{a} \& \mathbf{2 b})$.

Ethyl 2-[4-(2-Methylpropyl) phenyl] propanoate (ester of ibuprofen) (2a). Yield: $70.5 \%$, b.p.: $80-82^{\circ} \mathrm{C}, \mathrm{IR}\left(\mathrm{KBr}, \mathrm{cm}^{-1}\right): 2981$ (Ar-CH, str); 1733 (C=O ester, str); 1465, $1417\left(\mathrm{CH}_{3}-\mathrm{C}-\mathrm{H}\right.$, bend); 931, 875, 833 (Ar- $\mathrm{CH}$, bend).

2-[(2',6'-Dichlorophenyl) Amino] Phenylacetoxyacetate (Ester of Aceclofenac) (2b). Yield: $75 \%$, m.p.: $165-166^{\circ} \mathrm{C}$, IR (KBr, $\left.\mathrm{cm}^{-1}\right)$ : $3310(-\mathrm{NH}$, str $) ; 2914(\mathrm{Ar}-\mathrm{CH}$, str $) ; 1730(\mathrm{C}=\mathrm{O}$ ester, str); 1608 (NH, bend); 1238, 1166 (C-O, str); 783, 748 (Ar-CH, bend), $669(\mathrm{C}-\mathrm{Cl}$, bend $)$.

2.1.3. Synthesis of Hydrazides (3) [12]. Ester of respective NSAID (0.06 mole) and $98 \%$ hydrazine hydrate $(0.09$ mole) were refluxed in absolute ethanol $(50 \mathrm{~mL})$ for 300 minutes at 490 watt in microwave oven. The mixture was concentrated, cooled, and poured in ice cold water. The white solid thus separated, was filtered, dried, and recrystallized from absolute ethanol.

2-[4-(2-Methylpropyl) phenyl] propanehydrazide (hydrazide of ibuprofen) (3a). Yield: $87.38 \%$, m.p.: $60^{\circ} \mathrm{C}$, IR $\left(\mathrm{KBr}, \mathrm{cm}^{-1}\right)$ : 3280 (-NH, str); 3039, 2954, 2869 (Ar-CH, str); 1689, 1641
( $\mathrm{C}=\mathrm{O}, \quad$ str amide $), 1508,1460(-\mathrm{NH}$, bend $) ; 1417,1361$ $\left(\mathrm{CH}_{3}-\mathrm{C}-\mathrm{H}\right.$, bend); 846, 784, 729 (Ar-CH, bend).

2-[(2', 6'-Dichlorophenyl). amino] phenylacetoxyacetylhydrazide (hydrazide of aceclofenac) (3b). Yield: 81.78\%, m.p.: 190-196 ${ }^{\circ} \mathrm{C}$, IR $\left(\mathrm{KBr}, \mathrm{cm}^{-1}\right): 3323$ (-NH, str); 2923 ( $\mathrm{Ar}-\mathrm{CH}$, str); 1635 ( $\mathrm{C}=\mathrm{O}$, str, amide); $1507(\mathrm{NH}$, bend $) ; 773,746$ (Ar-CH, bend); 671, 557 (C-Cl, bend).

\subsubsection{Synthesis of 2,5-Disubstituted-1,3,4-oxadiazoles (6a-6j).}

(1) Method A: Conventional Method: By using phosphorous oxychloride $\left(\mathrm{POCl}_{3}\right)$ [13]. A mixture of hydrazide (3a or $\mathbf{3 b}$, 0.01 mole), various aromatic acid $(\mathbf{4 a}-\mathbf{4 j})(0.01 \mathrm{~mole})$, and phosphorous oxychloride $(15 \mathrm{~mL})$ was refluxed at $110-115^{\circ} \mathrm{C}$ for 7-8 h. It was then cooled to room temperature and neutralized with ice cold solution of $10 \%$ sodium bicarbonate. The extraction was done with dichloromethane $(2 \times 20 \mathrm{~mL})$ and the organic layer was separated, dried over anhydrous sodium sulphate and then evaporated. Reaction was monitored by TLC (Ethyl acetate: Hexane, 1:3). The compounds were further purified by column chromatography (Silica gel, Ethyl acetate: Hexane, $1: 3$ ) to yield pure products.

2-\{1-[4-(2-Methylpropyl) phenyl] ethyl\}-5-phenyl-1,3,4-oxadiazole (6a). Mol. Formula: $\mathrm{C}_{20} \mathrm{H}_{22} \mathrm{~N}_{2} \mathrm{O}$; Mol. Wt.: 306.40; $\mathrm{mp}\left({ }^{\circ} \mathrm{C}\right): 82-84^{\circ} \mathrm{C}$; IR $\left(\mathrm{KBr}, \mathrm{cm}^{-1}\right): 2923$ (Ar-CH, str); 1604 (C=N, str); 1278, 1093, 1012 (C-O-C, oxadiazole); 837, 744 (Ar-CH, bend).

${ }^{1} \mathrm{H}-\mathrm{NMR}(\delta, \mathrm{ppm}): 7.48-7.45$ (d, 2H, Ar-H); 7.26-7.23 (m, 5H, Ar-H); 7.13-7.10 (d, 2H, Ar-H); 4.42-4.40 (m, $1 \mathrm{H}$, $\mathrm{CH}) ; 2.46-2.43$ (d, $2 \mathrm{H}, \mathrm{CH}_{2}$ of Isobutyl group); $1.84-1.81$ (d, $\left.3 \mathrm{H}, \mathrm{CH}_{3}\right) ; 1.8-1.89$ (d, $1 \mathrm{H}, \mathrm{CH}$ of Isobutyl group); 0.90-0.88 (d, $6 \mathrm{H},\left(\mathrm{CH}_{3}\right)_{2}$ of Isobutyl group).

2-\{1-[4-(2-Methylpropyl) phenyl] ethyl\}-5-(4-methylphenyl)1,3,4-oxadiazole (6b). Mol. Formula: $\mathrm{C}_{21} \mathrm{H}_{24} \mathrm{~N}_{2} \mathrm{O}$; Mol. Wt.: 320.42; mp ( $\left.{ }^{\circ} \mathrm{C}\right): 79-80 ; \mathrm{IR}\left(\mathrm{KBr}, \mathrm{cm}^{-1}\right): 2954,2869$ ( $\mathrm{Ar}-\mathrm{CH}$, str); 1616, $1560(\mathrm{C}=\mathrm{N}$, str); 1182, 1118, 1066, $1004(\mathrm{C}-\mathrm{O}-\mathrm{C}$, oxadiazole); 825, 730 ( $\mathrm{Ar}-\mathrm{CH}$, bend).

2-\{1-[4-(2-methylpropyl) phenyl] ethyl\}-5-(2-hydroxyphenyl)-1,3,4-oxadiazole (6c). Mol. Formula: $\mathrm{C}_{20} \mathrm{H}_{22} \mathrm{~N}_{2} \mathrm{O}_{2}$; Mol. Wt.: 322.40; $\mathrm{mp}\left({ }^{\circ} \mathrm{C}\right): 256$ (dec.); IR $\left(\mathrm{KBr}, \mathrm{cm}^{-1}\right): 3461(-\mathrm{OH}$, str); 2918, 2848 (Ar-CH, str); 1614, $1558(\mathrm{C}=\mathrm{N}$, str); 1164, 1099, 1020 (C-O-C, oxadiazole); 848, 802, 742 (Ar-CH, bend).

${ }^{1} \mathrm{H}-\mathrm{NMR}(\delta, \mathrm{ppm}): 10.06(1 \mathrm{H}, \mathrm{OH}) ; 8.46(1 \mathrm{H}, \mathrm{Ar}-\mathrm{H}$ of aryl acid, $7.89(1 \mathrm{H}, \mathrm{Ar}-\mathrm{H}) ; 7.76(1 \mathrm{H}, \mathrm{Ar}-\mathrm{H}) ; 7.50(4 \mathrm{H}, \mathrm{Ar}-\mathrm{H}$ of ar yl acid moiety); $7.39(1 \mathrm{H}, \mathrm{Ar}-\mathrm{H}) ; 3.10(1 \mathrm{H}$, Aliphatic $\mathrm{CH}) ; 2.26(1 \mathrm{H}, \mathrm{CH}$ of Isobutyl group $) ; 2.20\left(2 \mathrm{H}, \mathrm{CH}_{2}\right.$ of Isobutyl group); $1.54\left(3 \mathrm{H}\right.$, Aliphatic $\left.\mathrm{CH}_{3}\right) ; 0.88\left(6 \mathrm{H},\left(\mathrm{CH}_{3}\right)\right.$ of Isobutyl group).

2-\{1-[4-(2-Methylpropyl) phenyl] ethyl\}-5-benzyl-1,3,4-oxadiazole (6d). Mol. Formula: $\mathrm{C}_{21} \mathrm{H}_{24} \mathrm{~N}_{2} \mathrm{O}$; Mol. Wt.: 320.42; mp $\left({ }^{\circ} \mathrm{C}\right): 81-83$; IR $\left(\mathrm{KBr}, \mathrm{cm}^{-1}\right): 3190,3055$ (Ar-CH, str); 1645, 1577 (C=N str); 1517, 1448 (C-str); 1226, 1159, 1095 (C-O-C oxadiazole); 850 (C-H, aliphatic); 746, 689 (Ar-CH, bend). 
Mass (m/e): $320\left(\mathrm{M}^{+}\right), 244(\mathrm{M}-77)$.

2-\{1-[4-(2-Methylpropyl) phenyl] ethyl\}-5-pyridyl-1,3,4oxadiazole (6e). Mol. Formula: $\mathrm{C}_{19} \mathrm{H}_{21} \mathrm{~N}_{3} \mathrm{O}$; Mol. Wt.: 307.39; mp ( $\left.{ }^{\circ} \mathrm{C}\right): 82-84$; IR $\left(\mathrm{KBr}, \mathrm{cm}^{-1}\right)$ : 3051 (Ar-CH, str); 1602 $(\mathrm{C}=\mathrm{N}, s t r) ; 1546$ (C-O, str); 1166, 1080, 1076 (C-O-C, oxadiazole); 814,700 (Ar-CH, bend).

2-[2-[(2',6'-Dichlorophenyl) amino] phenylacetoxymethyl]5-phenyl-1,3,4-oxadiazole (6f). Mol. Formula: $\mathrm{C}_{23} \mathrm{H}_{17} \mathrm{Cl}_{2}$ $\mathrm{N}_{3} \mathrm{O}_{3}$; Mol. Wt.: 454.30; $\mathrm{mp}\left({ }^{\circ} \mathrm{C}\right): 80-82$; IR $\left(\mathrm{KBr}, \mathrm{cm}^{-1}\right): 3305$ (isoH, str); 2923, 2852 (Ar-CH, str); 1683 (C=O, str); 1633 $(\mathrm{C}=\mathrm{N}$, str $) ; 1512(\mathrm{NH}$, bend $) ; 1359,1309$ (C-N, bend); 1217, 1080, 966 (C-O-C oxadiazole); 784,738 (Ar-CH, bend); 742, $694(\mathrm{C}-\mathrm{Cl}$, bend $)$.

2-[2-[(2',6'-Dichlorophenyl) amino] phenylacetoxymethyl]5-[4-methylphenyl]-1,3,4-oxadiazole (6g). Mol. Formula: $\mathrm{C}_{24} \mathrm{H}_{19} \mathrm{Cl}_{2} \mathrm{~N}_{3} \mathrm{O}_{3}$; Mol. Wt.: 468.33; mp ( $\left.{ }^{\circ} \mathrm{C}\right)$ : 85-86; IR ( $\mathrm{KBr}$, $\mathrm{cm}^{-1}$ ): 3280 (NH, str); 3068, 2923 (Ar-CH, str); 1691 (C=O, str); 1571, 1521 (NH, bend); 1346, 1307 (C-N, bend); 1151, 1105, 1010, 970 (C-O-C oxadiazole); 788, 740 (Ar-CH, bend); 611 (C-Cl, bend).

2-[2-[(2',6'-Dichlorophenyl) amino] phenylacetoxymethyl]5-[2-hydroxyphenyl]-1,3,4-oxadiazole (6h). Mol. Formula: $\mathrm{C}_{23} \mathrm{H}_{17} \mathrm{Cl}_{2} \mathrm{~N}_{3} \mathrm{O}_{4}$; Mol. Wt.: 470.30; mp ( $\left.{ }^{\circ} \mathrm{C}\right)$ : 128-130; IR $\left(\mathrm{KBr}, \mathrm{cm}^{-1}\right)$ : 3452 (-OH, str); 3305 (-NH, str); 3066, 2921 (Ar-CH, str); 1604, 1519 (NH, bend); 1151, 1105, 1012 C-O-C oxadiazole), 784, 738 (Ar- $\mathrm{CH}$, bend), 611 (C-Cl, bend).

${ }^{1} \mathrm{H}-\mathrm{NMR}(\delta, \mathrm{ppm}): 9.60(\mathrm{~s}, 1 \mathrm{H}, \mathrm{OH}) ; 7.90-7.80$ (d, 2H, Ar-H); 7.50-7.40 (d, 2H, Ar-H); 7.20-7.18 (d, 3H, Ar-H); 7.00-6.90 (d, 1H, Ar-H); 6.90-6.80 (d, 1H, Ar-H); 6.80-6.70 (d, 1H, Ar-); 5.10-5.00 (s, 2H, Aliphatic CH); 4.5 (s, 1H, NH); 3.6-3.5 (s, 2H, Aliphatic $\mathrm{CH})$.

2-[2-[(2',6'-Dichlorophenyl) amino] phenylacetoxymethyl]5-benzyl-1,3,4-oxadiazole (6i). Mol. Formula: $\mathrm{C}_{24} \mathrm{H}_{19} \mathrm{Cl}_{2}$ $\mathrm{N}_{3} \mathrm{O}_{3}$; Mol. Wt.: 468.33; mp $\left({ }^{\circ} \mathrm{C}\right)$ : 80-83; IR $\left(\mathrm{KBr}, \mathrm{cm}^{-1}\right)$ : 3060, 3029, 2954 (Ar-CH, str); 1730 (C=O, str); 1612 $(\mathrm{C}=\mathrm{N}$, str $) ; 1566$ (NH, bend); 1475, 1438 (C-O, str); 1311 (C-N, bend); 1199, 1157, 1105 (C-O-C oxadiazole). 790,746 (Ar-CH, bend); 696 (C-Cl, bend).

Mass (m/e): 467.1( $\left.\mathrm{M}^{+}\right), 391(\mathrm{M}-77)$.

2-[2-[(2',6'-Dichlorophenyl) amino] phenylacetoxymethyl]5-[4-pyridyl]-1,3,4-oxadiazole (6j). Mol. Formula: $\mathrm{C}_{22} \mathrm{H}_{16}$ $\mathrm{Cl}_{2} \mathrm{~N}_{4} \mathrm{O}_{3}$. Mol. Wt.: 455.29; $\mathrm{mp}\left({ }^{\circ} \mathrm{C}\right)$ : 85-86; IR $\left(\mathrm{KBr}, \mathrm{cm}^{-1}\right)$ : 3352 (NH, str); 3107 (Ar-CH, str); 1714, 1689 (C=O, str), $1598(\mathrm{C}=\mathrm{N}$, str $) ; 1500(\mathrm{NH}$, bend $), 848,730$ (Ar-CH, bend).

(2) Method B: Microwave-Assisted synthesis (alternate method) using lead oxide $\left(\mathrm{PbO}_{2}\right)$ [14, 15]. A mixture of hydrazide (3a or 3b, 0.005 mole) and aromatic aldehyde $(\mathbf{5} \mathbf{a}-5 \mathbf{j}, 0.005$ mole) was taken in $20 \mathrm{~mL}$ of acetic acid. The Microwave flask containing this mixture was heated at $70^{\circ} \mathrm{C}$ for $60 \mathrm{~min}$ at 560 watt under microwave radiation. The reaction mixture was then cooled to room temperature. Lead oxide $(1.0 \mathrm{~g})$ was added into it under stirring. The temperature was raised to $100^{\circ} \mathrm{C}$ and stirred at the same temperature for $120 \mathrm{~min}$ at 560 watt. It was then hot filtered to remove the $\mathrm{PbO}_{2}$ and allowed to attain the room temperature and then poured onto ice-cold water. The solid, thus separated was filtered, dried, and purified by column chromatography (Silica gel, Ethyl acetate/Hexane $1: 3$ ) to afford the compounds.

2-\{1-[4-(2-Methylpropyl) phenyl] ethyl\}-5-phenyl-1,3,4-oxadiazole (6a). Mol. Formula: $\mathrm{C}_{20} \mathrm{H}_{22} \mathrm{~N}_{2} \mathrm{O}$; Mol. Wt.: 306.40; mp $\left({ }^{\circ} \mathrm{C}\right)$ : 82-84; IR (KBr, cm ${ }^{-1}$ ): 2954, 2867 (Ar-CH, str); 1683 $(\mathrm{C}=\mathrm{O}$, str $) ; 1606(\mathrm{C}=\mathrm{N}$, str $), 1508(\mathrm{~N}-\mathrm{H}$, bend $), 1278,1093$, 1012 (C-O-C, oxadiazole), 837, 744 (Ar-CH, bend).

2-\{1-[4-(2-Methylpropyl) phenyl] ethyl\}-5-(4-methylphenyl)1,3,4-oxadiazole (6b). Mol. Formula: $\mathrm{C}_{21} \mathrm{H}_{24} \mathrm{~N}_{2} \mathrm{O}$; Mol. Wt.: 320.42; mp ( $\left.{ }^{\circ} \mathrm{C}\right)$ : 77-80; IR $\left(\mathrm{KBr}, \mathrm{cm}^{-1}\right)$ : 2954 ( $\left.\mathrm{Ar}-\mathrm{CH}, \mathrm{str}\right)$; 1602 (C=N, str); 1558, 1515, 1485 (C-O, str); 1068 (C-O-C, oxadiazole).

2-\{1-[4-(2-methylpropyl) phenyl] ethyl\}-5-(2-hydroxyphenyl)-1,3,4-oxadiazole (6c). Mol. Formula: $\mathrm{C}_{20} \mathrm{H}_{22} \mathrm{~N}_{2} \mathrm{O}_{2}$; Mol. Wt.: 322.40; mp ( $\left.{ }^{\circ} \mathrm{C}\right): 260$ (dec.); IR $\left(\mathrm{KBr}, \mathrm{cm}^{-1}\right): 3357(\mathrm{OH}$, str); 3261 ( NH, str); 3068, 2900 (Ar-CH, str); 1571, 1517 (NH, bend); 1153, 1108, 1010, (C-O-C oxadiazole); 784, 738 (Ar-CH, bend).

2-\{1-[4-(2-Methylpropyl) phenyl] ethyl\}-5-benzyl-1,3,4-oxadiazole (6d). Mol. Formula: $\mathrm{C}_{21} \mathrm{H}_{24} \mathrm{~N}_{2} \mathrm{O}$; Mol. Wt.: 320.42; mp $\left({ }^{\circ} \mathrm{C}\right)$ : 81-83; IR (KBr, cm $\left.{ }^{-1}\right): 3072,2916,2848$ (Ar-CH, str), $1660(\mathrm{C}=\mathrm{N}$, str $), 1556,1473$ (NH, bend); 1438 (O-H, bend), 1309, 1228 (C-O-C oxadiazole), 790, 742 (Ar-CH, bend).

2-\{1-[4-(2-methylpropyl) phenyl] ethyl\}-5-pyridyl-1,3,4-oxadiazole (6e). Mol. Formula: $\mathrm{C}_{19} \mathrm{H}_{21} \mathrm{~N}_{3} \mathrm{O}$; Mol. Wt.: 307.39; $\mathrm{mp}\left({ }^{\circ} \mathrm{C}\right): 80-83$; IR $\left(\mathrm{KBr}, \mathrm{cm}^{-1}\right)$ : 3074, 3053, 2925 (Ar-CH, str); 1564, 1521 ( $\mathrm{NH}$, bend); 1475,1440 (C=N, str); 1201, 1147, 1101, 1010, 972 (C-O-C oxadiazole); 777, 740 (Ar-CH, bend).

${ }^{1} \mathrm{H}-\mathrm{NMR}(\delta, \mathrm{ppm}): 8.76(2 \mathrm{H}, \mathrm{Ar}-\mathrm{H}(\alpha-\mathrm{H})) ; 7.86(2 \mathrm{H}$, $\operatorname{Ar}-\mathrm{H}(\beta-\mathrm{H})) ; 7.23(4 \mathrm{H}, \mathrm{Ar}-\mathrm{H}) ; 4.43(1 \mathrm{H}$, Aliphatic $\mathrm{CH}) ; 2.43$ (2H, $\mathrm{CH}_{2}$ of Isobutyl group); 1.81 ( $1 \mathrm{H}, \mathrm{CH}$ of Isobutyl group); $1.50\left(3 \mathrm{H}\right.$, Aliphatic $\left.\mathrm{CH}_{3}\right) ; 0.88\left(6 \mathrm{H},\left(\mathrm{CH}_{3}\right)_{2}\right.$ of Isobutyl group).

2-[2-[(2',6'-Dichlorophenyl) amino] phenylacetoxymethyl]5-phenyl-1,3,4-oxadiazole (6f). Mol. Formula: $\mathrm{C}_{23} \mathrm{H}_{17}$ $\mathrm{Cl}_{2} \mathrm{~N}_{3} \mathrm{O}_{3}$; Mol. Wt.: 454.30; mp ( $\left.{ }^{\circ} \mathrm{C}\right)$ : 79-81; IR $\left(\mathrm{KBr}, \mathrm{cm}^{-1}\right)$ : 3217 (NH, str); 3068, 2923, 2852 (Ar-CH, str); $1564(\mathrm{NH}$, bend); 1473, 1440 (C-O, str) 1205, 1103, 1010 (C-O-C oxadiazole), 784, 738 (Ar-CH, bend), 611 (C-Cl, bend).

2-[2-[(2',6'-Dichlorophenyl) amino] phenylacetoxymethyl]5-[4-methylphenyl]-1,3,4-oxadiazole (6g). Mol. Formula: $\mathrm{C}_{24} \mathrm{H}_{19} \mathrm{Cl}_{2} \mathrm{~N}_{3} \mathrm{O}_{3}$; Mol. Wt.: 468.33; mp ( $\left.{ }^{\circ} \mathrm{C}\right)$ : 85-86; IR ( $\mathrm{KBr}$, $\left.\mathrm{cm}^{-1}\right)$ : $3305(\mathrm{NH}$, str $) ; 3064(\mathrm{Ar}-\mathrm{CH}$, str $) ; 1672(-\mathrm{C}=\mathrm{O}$, 
str); 1604, 1533 (NH, bend), 1255, 1170, 1068, 970 (C-O-C oxadiazole), 744, 688 (Ar-CH, bend), 611 (C-Cl, bend).

${ }^{1} \mathrm{H}-\mathrm{NMR}(\delta, \mathrm{ppm}):$ 7.54-7.52 (d, 2H, Ar-H); 7.45-7.42 (d, 1H, Ar-H); 7.40-7.39 (d, 1H, Ar-H); 7.21-7.16 (m, 5H, Ar-H); 6.90-6.80 (t, 1H, Ar-H); 6.66-6.70 (d, 1H, Ar-H); $5.00\left(\mathrm{~s}, 2 \mathrm{H}\right.$, Aliphatic $\left.\mathrm{CH}_{2}\right) ; 4.80-4.75(\mathrm{~s}, 1 \mathrm{H}, \mathrm{NH}) ; 3.22-3.20$ (s, 2H, Aliphatic $\mathrm{CH}_{2}$ ).

2-[2-[(2',6'-Dichlorophenyl) amino] phenylacetoxymethyl]5- [2-hydroxyphenyl]-1,3,4-oxadiazole (6h). Mol. Formula: $\mathrm{C}_{23} \mathrm{H}_{17} \mathrm{Cl}_{2} \mathrm{~N}_{3} \mathrm{O}_{4}$; Mol. Wt.: 470.30; mp ( $\left.{ }^{\circ} \mathrm{C}\right)$ : 129-132; IR $\left(\mathrm{KBr}, \mathrm{cm}^{-1}\right)$ : $3452(-\mathrm{OH}$, str $) ; 3305$ (-NH, str); 3066, 2921 (Ar-CH, str); 1604, 1519 (NH, bend); 1151, 1105, 1012 (C-O-C oxadiazole); 784,738 (Ar-CH, bend); 611 (C-Cl, bend).

2-[2-[(2',6'-Dichlorophenyl) amino] phenylacetoxymethyl]5-benzyl-1,3,4-oxadiazole (6i). Mol. Formula: $\mathrm{C}_{24} \mathrm{H}_{19} \mathrm{Cl}_{2}$ $\mathrm{N}_{3} \mathrm{O}_{3}$; Mol. Wt.: 468.33; mp $\left({ }^{\circ} \mathrm{C}\right): 80-83$; IR $\left(\mathrm{KBr}, \mathrm{cm}^{-1}\right)$ : 3072, $2972(\mathrm{C}-\mathrm{H}, s t r) ; 1639(\mathrm{C}=\mathrm{O}, s t r) ; 1598,1555(\mathrm{C}=\mathrm{N}$, str); 1190, 1112, 1074, (C-O-C, oxadiazole); 891, 846 (C-H, aliphatic); 738 (Ar-CH, bend).

Mass (m/e): 467.1( $\left.\mathrm{M}^{+}\right), 391(\mathrm{M}-77)$.

2-[2-[(2',6'-Dichlorophenyl) amino] phenylacetoxymethyl]5-[4-pyridyl]-1,3,4-oxadiazole (6j). Mol. Formula: $\mathrm{C}_{22} \mathrm{H}_{16}$ $\mathrm{Cl}_{2} \mathrm{~N}_{4} \mathrm{O}_{3}$; Mol. Wt.: 455.29; mp ( $\left.{ }^{\circ} \mathrm{C}\right)$ : 83-86; IR $\left(\mathrm{KBr}, \mathrm{cm}^{-1}\right)$ : 3282 (N-H, bend); 3033, 2921 (Ar-CH, str); $1666(\mathrm{C}=\mathrm{O}$, str), $1625(\mathrm{C}=\mathrm{N}$, str $), 1581,1529(\mathrm{~N}-\mathrm{H}$, bend $) ; 1095,1049$, 985 (C-O-C oxadiazole); 896, 781 (Ar-CH, bend); 608, 636 (C-Cl).

${ }^{1} \mathrm{H}-\mathrm{NMR}(\delta, \mathrm{ppm}): 8.98(2 \mathrm{H}, \mathrm{Ar}-\mathrm{H}(\alpha-\mathrm{H})) ; 8.70(1 \mathrm{H}$, Ar-H); 8.31 (1H, Ar-H); $8.06(2 \mathrm{H}, \operatorname{Ar}-\mathrm{H}(\beta-\mathrm{H})) ; 7.69$ $(1 \mathrm{H}, \mathrm{Ar}-\mathrm{H}) ; 7.56(2 \mathrm{H}, \mathrm{Ar}-\mathrm{H}) ; 6.86(1 \mathrm{H}, \mathrm{Ar}-\mathrm{H}) ; 4.88(2 \mathrm{H}$, Aliphatic $\left.\mathrm{CH}_{2}\right) ; 4.05(1 \mathrm{H}, \mathrm{NH}) ; 3.49\left(2 \mathrm{H}\right.$, Aliphatic $\left.\mathrm{CH}_{2}\right)$.

\subsection{Results and Discussion}

2.2.1. Chemistry. A novel series of NSAIDs-based 1,3,4oxadiazoles was synthesized using two methods (Method A and Method B). Method A was conventional method and Method B was based on microwave-assisted synthesis using novel catalytic reagent. The basic theme in these synthetic strategies was to synthesize NSAID-based pharmacologically important 1,3,4-oxadiazoles in economic and rapid manner. The aim was successfully achieved by developing a convenient, rapid, and cost-effective method.

The acid hydrazides $(\mathbf{3} \mathbf{a} / \mathbf{3 b})$ were prepared by esterification of $(\mathbf{1} \mathbf{a} / \mathbf{1} \mathbf{b})$, followed by the treatment of each separately with hydrazine hydrate in absolute ethanol. Various 2-aryl substituted-1,3,4-oxadiazole derivatives (6a-6j) were further synthesized using both methods. The purity of synthesized compounds was established by TLC using appropriate solvent system. They were characterized by IR, ${ }^{1} \mathrm{H}$ NMR, and mass spectroscopic techniques. The appearance of $-\mathrm{C}=\mathrm{O}$ str at $>1730 \mathrm{~cm}^{-1}$ in IR spectrum showed the formation of ester. The hydrazide formation was also shown by IR spectra as the $-\mathrm{C}=\mathrm{O}$ str, amide was seen around $1635-1650 \mathrm{~cm}^{-1}$.
Strong peaks in the range of $1250-1000 \mathrm{~cm}^{-1}$ attributed to oxadiazole ring. Few of these compounds were further confirmed by their mass spectra.

2.2.2. Comparison between Synthesis by Conventional (Method A) and Alternate Methods (Method B). A comparison of these two methods was carried out on the basis of fundamental principles and concepts of green chemistry.

(1) Yield and Purity. Newly synthesized oxadiazole compounds, their yields, and physical characteristics are compared in Tables 1 and 2. From these tables, it becomes apparent that yields of compounds obtained by cost-effective alternate route (Method B) were slightly higher (10-15\% more) than the conventional route (Method A). However, as desired, their physical constants remain the same by both methods.

(2) Reaction Time. This approach shortened the reaction time significantly. In Method A, 8-9 h were required to yield the desired product, whereas Method $\mathrm{B}$ required maximum $3 \mathrm{~h}$ to complete the same reaction. Hence, Method B can be considered as faster than the Method A with respect to time.

(3) Minimization of Energy Consumption. It is observed that Method B increases the yield and decreases the duration of the time required for the reaction. The use of microwave helped to reduce the thermal and mechanical energies. It also helped to reduce the wastage of other resources; for example, continuous $8 \mathrm{~h}$ water supply for the reaction was required in Method A, while Method B required only $3 \mathrm{~h}$ to give the same desired product/s.

Hence, reducing the unnecessary usage of important resources like water, electricity resulted in minimization of energy consumption.

(4) Environmental Quotient. Effect of chemicals on the surrounding environment is one of the important aspects of the ecofriendly and reliable chemistry. In Method A, the reagent used $\left(\mathrm{POCl}_{3}\right)$ is hazardous and requires precaution in handling. Also, $\mathrm{HCl}$ gas which evolves during the reaction is toxic to environment and to human health. Hence, the use of $\mathrm{POCl}_{3}$ is not considered as ecofriendly. On other side, $\mathrm{PbO}_{2}$ (Method B) increases the conversion and selectivity of the reaction.

Considering all the previous factors, it can be firmly said that Method B is economic, safe, and rapid synthetic method to synthesize the compounds mentioned herein.

\section{Evaluation of Antimicrobial and Analgesic Activity}

\subsection{Biological Evaluation}

3.1.1. Antimicrobial Activity [16-18]. The newly synthesized compounds were tested in vitro for their antimicrobial activity against bacteria like $S$. aureus, $E$. coli and fungi like $C$. albicans, $A$. niger. Minimum inhibitory concentration (MIC) 
TABLE 1: Physical characteristics of compounds (6a-6j) synthesized by using Method A (conventional method).

\begin{tabular}{|c|c|c|c|c|c|c|c|c|c|c|}
\hline \multirow{2}{*}{ Comp. Number } & \multicolumn{10}{|c|}{ Method A (conventional method) } \\
\hline & $6 a$ & $6 \mathbf{b}$ & $6 c$ & $6 d$ & $6 e$ & $6 f$ & $6 \mathrm{~g}$ & $6 \mathbf{h}$ & $6 \mathbf{i}$ & $6 \mathbf{j}$ \\
\hline Yield (\%) & 84.29 & 86.11 & 81 & 87 & 85.33 & 83.2 & 82 & 84.5 & 81 & 83.46 \\
\hline m.p. $\left({ }^{\circ} \mathrm{C}\right)$ & $82-84$ & $79-80$ & 256 (dec.) & $81-83$ & $82-84$ & $80-82$ & $85-86$ & $128-130$ & $80-83$ & $85-86$ \\
\hline $\mathrm{R} f$ & 0.74 & 0.80 & 0.76 & 0.79 & 0.78 & 0.76 & 0.78 & 0.80 & 0.82 & 0.85 \\
\hline
\end{tabular}

TABLE 2: Physical characteristics of compounds (6a-6j) synthesized by using Method B (alternate method).

\begin{tabular}{|c|c|c|c|c|c|c|c|c|c|c|}
\hline \multirow{2}{*}{ Comp Number } & \multicolumn{10}{|c|}{ Method B (alternate method) } \\
\hline & $6 a$ & $6 \mathbf{b}$ & $6 c$ & $6 d$ & $6 e$ & $6 f$ & $6 \mathrm{~g}$ & $6 h$ & $6 \mathbf{i}$ & $6 j$ \\
\hline Yield (\%) & 89.78 & 90.33 & 88.23 & 88.30 & 92 & 91.73 & 85.42 & 87 & 83.9 & 90.08 \\
\hline m.p. $\left({ }^{\circ} \mathrm{C}\right)$ & $82-84$ & $77-80$ & 260 (dec.) & $81-83$ & $80-83$ & $79-81$ & $85-86$ & $129-132$ & $80-83$ & $83-86$ \\
\hline $\mathrm{Rf}$ & 0.73 & 0.79 & 0.76 & 0.78 & 0.78 & 0.75 & 0.78 & 0.80 & 0.81 & 0.86 \\
\hline
\end{tabular}

TABLE 3: Antibacterial activity of compounds $\mathbf{6 a}-\mathbf{6 j}$.

\begin{tabular}{lccc}
\hline Number & Compound & $\begin{array}{c}\text { Minimum inhibitory concentration } \\
(\mathrm{MIC})(\mu \mathrm{g} / \mathrm{mL})\end{array}$ & E. coli \\
\hline 1 & & S. aureus & 62.5 \\
2 & $\mathbf{6 a}$ & 1 & 1 \\
3 & $\mathbf{6 b}$ & 2 & 125 \\
4 & $\mathbf{6 c}$ & 1 & 62.5 \\
5 & $\mathbf{6 d}$ & 4 & 62.5 \\
6 & $\mathbf{6 e}$ & 4 & 1 \\
7 & $\mathbf{6 f}$ & 4 & 1 \\
8 & $\mathbf{6 g}$ & 1 & 1 \\
9 & $\mathbf{6 h}$ & 2 & 1 \\
10 & $\mathbf{6 i}$ & 1 & 1 \\
11 & $\mathbf{6 j}$ & 1 & 1 \\
\hline
\end{tabular}

for antibacterial activity of all the synthesized compounds was determined by agar plate method. The standard used for the study was Ciprofloxacin. The results for antibacterial activity are reported in Table 3 . These compounds showed comparable activity with that of standard. Compounds with Aceclofenac backbone (6f-6j) exhibited excellent antibacterial activity against both tested bacteria, whereas Ibuprofen derivatives $(\mathbf{6 a}-\mathbf{6 e})$ were found to be selective against $S$. aureus, a gram-positive bacteria.

These compounds were also screened in vitro for their antifungal activity against $C$. albicans, A. niger using agar plate technique and fluconazole as standard. The screening results for antifungal activity are given in Table 4, which indicates that all compounds exhibit moderate to excellent activity against C. albicans and A. niger, respectively.

\subsubsection{Pharmacological Evaluation}

(1) Acute Toxicity [19]. Acute toxicity studies were conducted according to the OECD Guideline 425 using Up and Down
TABLE 4: Antifungal activity of compounds $\mathbf{6 a}-\mathbf{6 j}$.

\begin{tabular}{lccc}
\hline Number & Compound & $\begin{array}{c}\text { Minimum inhibitory concentration } \\
(\mathrm{MIC})(\mu \mathrm{g} / \mathrm{mL}) \\
\text { C. albicans }\end{array}$ & A.niger \\
\hline 1 & $\mathbf{6 a}$ & 8 & 16 \\
2 & $\mathbf{6 b}$ & 2 & 4 \\
3 & $\mathbf{6 c}$ & 8 & 8 \\
4 & $\mathbf{6 d}$ & 4 & 1 \\
5 & $\mathbf{6 e}$ & 1 & 31.25 \\
6 & $\mathbf{6 f}$ & 16 & 16 \\
7 & $\mathbf{6 g}$ & 16 & 1 \\
8 & $\mathbf{6 h}$ & 16 & 8 \\
9 & $\mathbf{6 i}$ & 8 & 4 \\
10 & $\mathbf{6 j}$ & 8 & 8 \\
11 & Fluconazole & 16 & \\
\hline
\end{tabular}

TABLE 5: $\mathrm{LD}_{50}$ values of compounds $\mathbf{6 a - 6 j}$.

\begin{tabular}{lc}
\hline Compound & $\mathrm{LD}_{50}(\mathrm{mg} / \mathrm{kg})$ \\
\hline $\mathbf{6 a}$ & 550 \\
$\mathbf{6 b}$ & 550 \\
$\mathbf{6 c}$ & 750 \\
$\mathbf{6 d}$ & 750 \\
$\mathbf{6 e}$ & 1750 \\
$\mathbf{6 f}$ & 550 \\
$\mathbf{6 g}$ & 750 \\
$\mathbf{6 h}$ & 550 \\
$\mathbf{6 i}$ & 1750 \\
$\mathbf{6 j}$ & 1750 \\
\hline
\end{tabular}

procedure. Swiss albino mice (female) weighing between 20 and $30 \mathrm{~g}$ were given orally a particular dose say $175 \mathrm{mg} / \mathrm{kg}$ and observed for a period of $48 \mathrm{hrs}$ for any mortality. The subsequent doses were then increased or decreased by a progression factor depending on its lethality. From Table 5, 
TABLE 6: Analgesic activity of compounds $\mathbf{6 a - 6 j}$.

\begin{tabular}{cccc}
\hline Compound & $\begin{array}{c}\text { Dose } \\
(\mathrm{mg} / \mathrm{kg})\end{array}$ & $\begin{array}{c}\text { Number of writhes in } 10 \text { min } \\
\text { after } \\
\text { treatment (mean } \pm \text { SEM })\end{array}$ & $\begin{array}{c}\% \\
\text { Inhibition }\end{array}$ \\
\hline Control & - & $32.8333 \pm 1.249444$ & 0.00 \\
Ibuprofen & 20 & $13.000 \pm 0.5163978^{* * *}$ & 60.045 \\
6a & 55 & $18.16667 \pm 1.137737^{* * *}$ & 44.50667 \\
$\mathbf{6 b}$ & 55 & $16.3333 \pm 0.9189366^{* * *}$ & 49.950 \\
$\mathbf{6 c}$ & 75 & $18.83333 \pm 0.7923243^{* * *}$ & 42.318 \\
$\mathbf{6 d}$ & 75 & $20.16667 \pm 0.4772607^{* * *}$ & 38.25333 \\
$\mathbf{6 e}$ & 175.0 & $8.3333 \pm 0.421637^{* * *}$ & 74.49333 \\
Control & - & $35.200 \pm 1.157584$ & 0.00 \\
Aceclofenac & 20 & $7.166667 \pm 0.6009252^{* * *}$ & 79.58833 \\
$\mathbf{6 f}$ & 55 & $24.66667 \pm 0.4944133^{* * *}$ & 29.215 \\
$\mathbf{6 g}$ & 75 & $19.66667 \pm 0.6666^{* * *}$ & 44.02833 \\
$\mathbf{6 h}$ & 55 & $11.16667 \pm 0.6009252^{* * *}$ & 66.92667 \\
$\mathbf{6 i}$ & 175.0 & $23.66667 \pm 0.9189366^{* * *}$ & 32.68833 \\
$\mathbf{6 j}$ & 175.0 & $11.16667 \pm 0.6009252^{* * *}$ & 68.055 \\
\hline
\end{tabular}

Data analyzed by one-way ANOVA followed by Dunnett's MultipleComparison Test $(n=6),{ }^{* * *} P<0.05$ significant from control.

the $\mathrm{LD}_{50}$ value of majority of the compounds was found to be more than $500 \mathrm{mg} / \mathrm{Kg}$, which make them safe for oral administration. One tenth dose of this value was selected as the therapeutic dose for the further evaluation of peripheral analgesic activity.

(2) Analgesic Activity $[20,21]$. Peripheral analgesic activity of the compounds $(\mathbf{6} \mathbf{a}-\mathbf{6 j})$ was performed by using acetic acid-induced writhing test in mice. The analgesic activity was evaluated using Ibuprofen and Aceclofenac as standards. The results emphasized that all compounds possess significant analgesic activity. The analgesic effect of compound $\mathbf{6 e}$ was found to be even better than Ibuprofen, while compounds $\mathbf{6 h}$ and $\mathbf{6 j}$ showed comparable activity with standard Aceclofenac. Data analyzed by one-way ANOVA followed by Dunnett's Multiple-Comparison Test $(n=6),{ }^{* * *} P<0.05$ significant from control, is given in Table 6.

\section{Conclusion}

Ideally chemical reactions should proceed with quantitative yield, low costs, and generating no waste. The use of techniques like microwave irradiation and proper catalytic reagents will impact greatly on the field of medicinal chemistry. Implementation of this in the pharmaceutical and medicinal chemistry sector will lead to inexpensive drugs and sustainable development of both human and surrounding. This will ultimately result in improved quality of life for the benefit of human welfare.

This study opens up a new area of cost-effective synthesis of biologically active 2,5-disubstituted-1,3,4-Oxadiazole compounds. The further investigation in terms of largescale synthesis and industrial utility of this method could be evaluated.

\section{References}

[1] M. Kidwai and R. Mohan, "Eco-friendly synthesis of antifungal azoles," Journal of Korean Chemical Society, vol. 48, no. 2, pp. 177-181, 2004.

[2] V. Polshettiwar and R. S. Varma, "Greener and expeditious synthesis of bioactive heterocycles using microwave irradiation," Pure and Applied Chemistry, vol. 80, no. 4, pp. 777-790, 2008.

[3] M. Kidwai and R. Mohan, "Green chemistry: an innovative technology," Foundations of Chemistry, vol. 7, no. 3, pp. 269-287, 2005.

[4] R. R. Somani and D. T. Makhija, "Improvement of GI tolerance of NSAIDs using oral prodrug approach," Der Pharmacia Lettre, vol. 2, no. 2, pp. 300-309, 2010.

[5] M. Amir and S. Kumar, "Synthesis and evaluation of antiinflammatory, analgesic, ulcerogenic and lipid peroxidation properties of ibuprofen derivatives," Acta Pharmaceutica, vol. 57, no. 1, pp. 31-45, 2007.

[6] A. Husain, A. Ahmad, M. M. Alam, M. Ajmal, and P. Ahuja, "Fenbufen based 3-[5-(substituted aryl)-1,3,4-oxadiazol-2-yl]1-(biphenyl-4-yl)propan-1-ones as safer antiinflammatory and analgesic agents," European Journal of Medicinal Chemistry, vol. 44, no. 9, pp. 3798-3804, 2009.

[7] R. R. Somani and P. Y. Shirodkar, "Synthesis, antibacterial and antitubercular evaluation of some 1,3,4-oxadiazole analogues," Asian Journal of Chemistry, vol. 20, no. 8, pp. 6189-6194, 2008.

[8] A. Mohammad and S. Kumar, "Synthesis and evaluation of antiinflammatory, analgesic, ulcerogenic and lipid peroxidation properties of ibuprofen derivatives," Acta Pharmaceutica, vol. 57, no. 1, pp. 31-45, 2007.

[9] R. R. Somani and P. Y. Shirodkar, "Oxadiazole: a biologically important heterocycle," Der Pharma Chemica, vol. 1, no. 1, pp. 130-140, 2009.

[10] M. Dabiri, P. Salehi, M. Baghbanzadeh, and M. Bahramnejad, "Alum $\left(\mathrm{KAl}\left(\mathrm{SO}_{4}\right)_{2} \cdot 12 \mathrm{H}_{2} \mathrm{O}\right)$ : an efficient and inexpensive catalyst for the one-pot synthesis of 1,3,4-oxadiazoles under solvent-free conditions," Monatshefte fur Chemie, vol. 138, no. 12, pp. 1253-1255, 2007.

[11] B. Furniss, A. H. Hannaford, P. W. G. Smith, and A. R. Tatchell, Vogel's Text book of Practical Organic Chemistry, Addison Wesley Longman, 5th edition, 1998.

[12] M. Amir and S. Kumar, "Synthesis and anti-inflammatory, analgesic, ulcerogenic and lipid peroxidation activities of some new 2-[(2,6-dichloroanilino) phenyl]acetic acid derivatives," European Journal of Medicinal Chemistry, vol. 39, no. 6, pp. 535-545, 2004.

[13] K. M. Khan, Z.-U. Rani, S. Perveen et al., "Microwave-assisted synthesis of 2,5- disubstituted-1, 3, 4-oxadiazoles," Letters in Organic Chemistry, vol. 1, pp. 50-52, 2004.

[14] M. D. Mullican, M. W. Wilson, D. T. Connor, C. R. Kostlan, D. J. Schrier, and R. D. Dyer, "Design of 5-(3,5-Di-tert-butyl4-hydroxyphenyl)-1,3,4-thiadiazoles, -1,3,4-oxadiazoles, and 1,2,4-triazoles as orally-active, nonulcerogenic antiinflammatory agents," Journal of Medicinal Chemistry, vol. 36, no. 8, pp. 1090-1099, 1993.

[15] R. Milcent and G. Barbier, “Oxydation d'hydrazones par'le bioxyde' de'plomb: nouvelles synthèses d'oxadiazoles-1,3,4 et de dérivés de l'amino-4-triazol-1,2,4 one-5," Journal Heterocyclic Chemistry, vol. 20, no. 1, pp. 77-80, 1983. 
[16] European Society of Clinical Microbiology and Infectious Diseases, "Determination of minimum inhibitory concentrations (MICs) of antibacterial agents by agar dilution," Clinical Microbiology and Infection, vol. 6, no. 9, pp. 509-515, 2000.

[17] K. C. Ravindra, H. M. Vagdevi, V. P. Vaidya, and B. Padmashali, "Synthesis, antimicrobial and antiinflammatory activities of 1,3,4-oxadiazoles linked to naphtho[2,1-b]furan," Indian Journal of Chemistry B, vol. 45, no. 11, pp. 2506-2511, 2006.

[18] S. G. Patil, M. Girisha, J. Badiger, S. M. Kudari, and M. G. Purohit, "Synthesis and anticonvulsant, antimicrobial activity of some bis-1,3,4-oxadiazole and bis-1,2,4-triazole derivatives from sebacic acid," Indian Journal of Heterocyclic Chemistry, vol. 17, no. 1, pp. 37-40, 2007.

[19] OECD [Organization for Economic Co-operation and Development], "Guideline 425: acute oral toxicity-Modified up and down procedure," Paris, 1998, http://www.oecd.org/.

[20] S. V. Bhandari, K. G. Bothara, M. K. Raut, A. A. Patil, A. P. Sarkate, and V. J. Mokale, "design, synthesis and evaluation of anti-inflammatory, analgesic and ulcerogenicity studies of novel S- substituted phenacyl-1,3,4-oxadiazole-2- thiol and schiff bases of diclofenac acid as nonulcerogenic derivatives," Bioorganic and Medicinal Chemistry, vol. 16, no. 4, pp. 1822-1831, 2008.

[21] H. G. Vogal, Drug Discovery and Evaluation Pharmacological Assay, 2nd edition, 2002. 

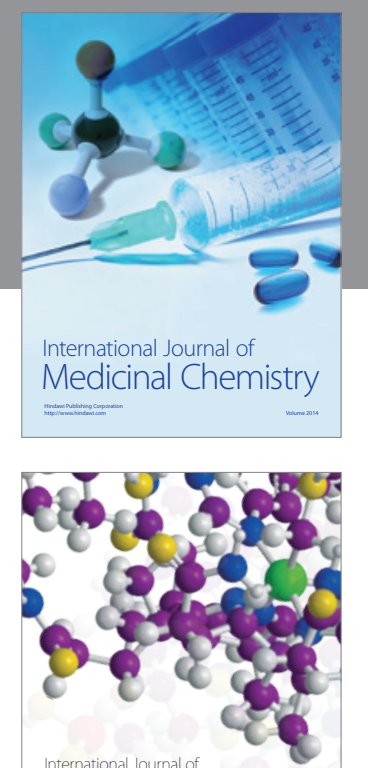

\section{Carbohydrate} Chemistry

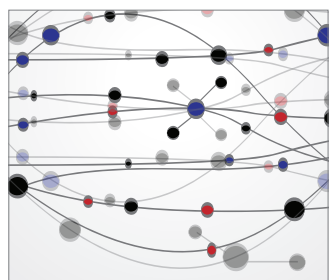

The Scientific World Journal
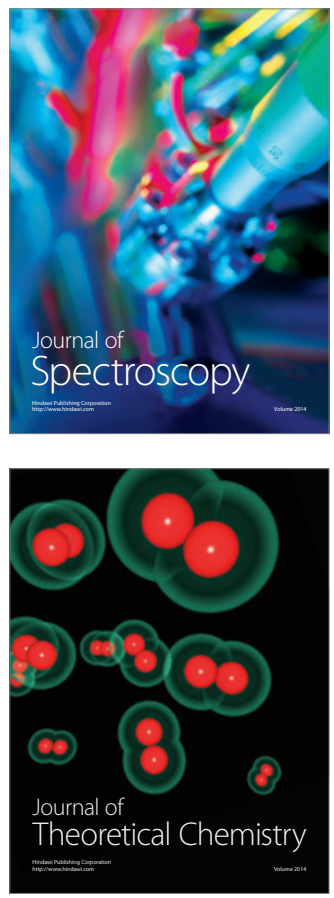
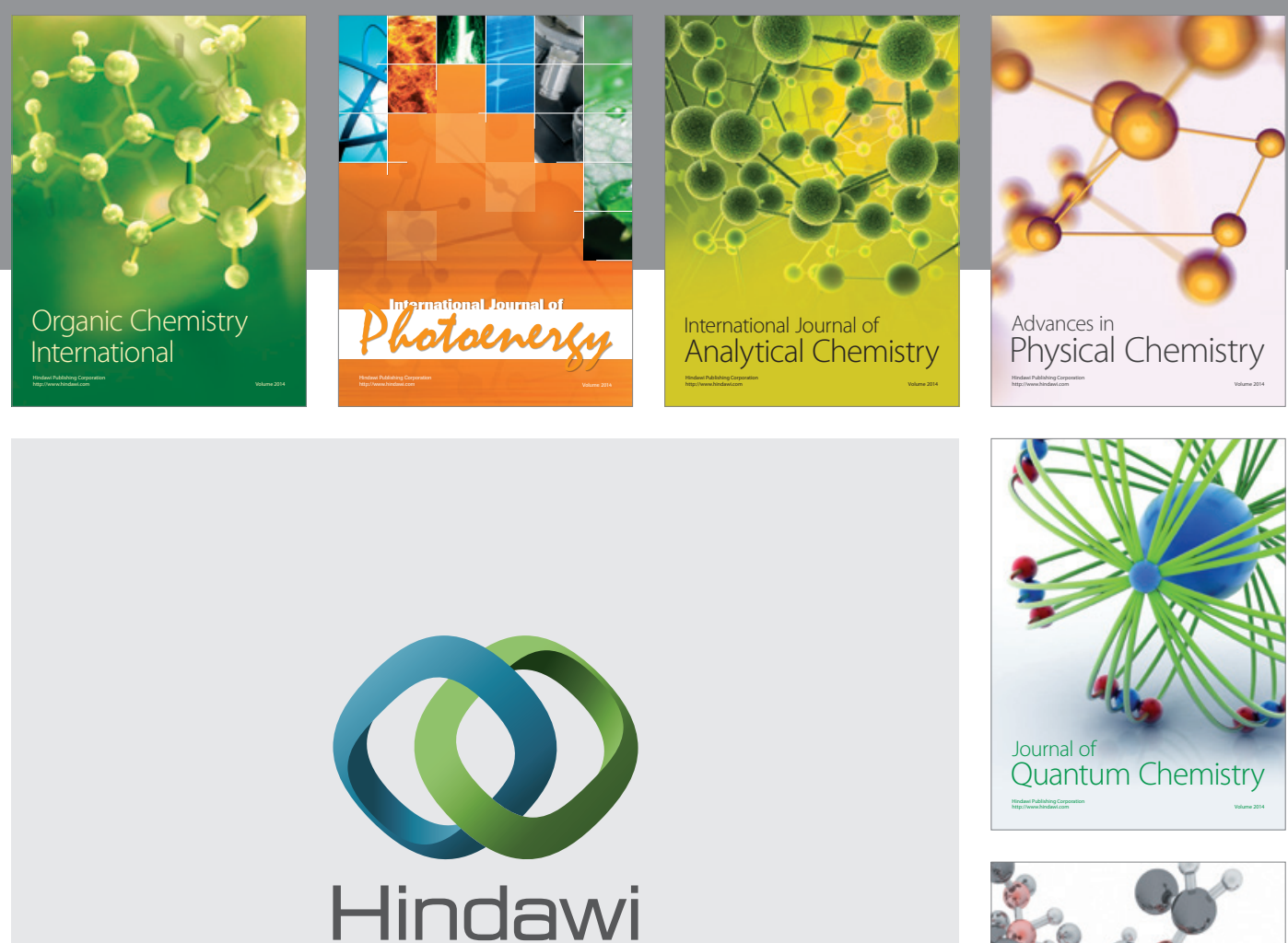

Submit your manuscripts at

http://www.hindawi.com

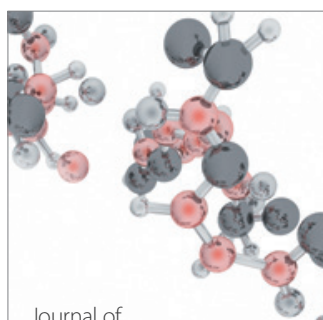

Analytical Methods

in Chemistry

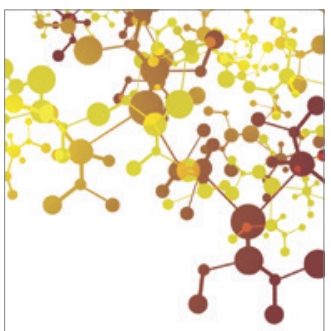

Journal of

Applied Chemistry

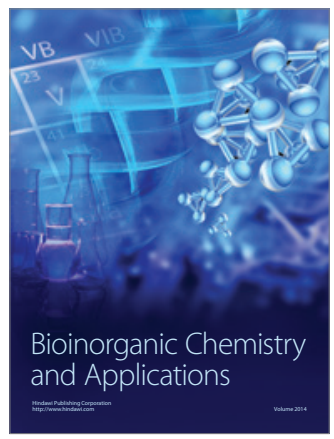

Inorganic Chemistry
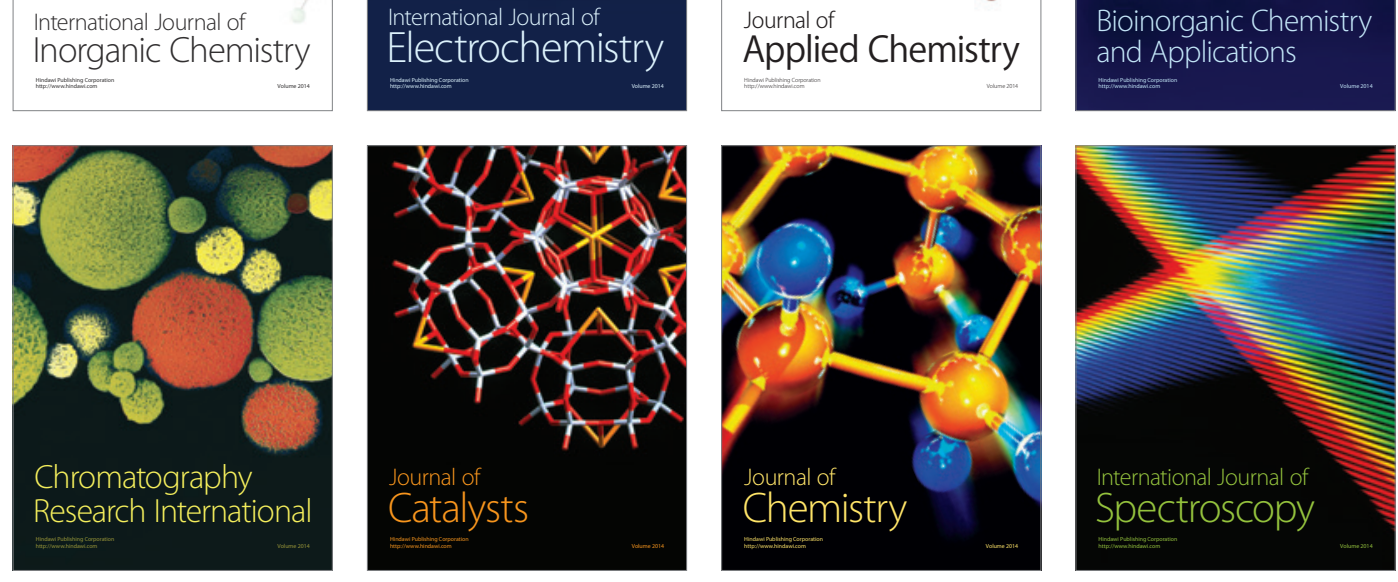